Wien klin Mag 2021 $\cdot 24: 20-33$ https://doi.org/10.1007/s00740-020-00379-5 Online publiziert: 30 . November 2020 (c) Der/die Autor(en) 2020

\author{
Almut Böer-Auer ${ }^{1,2}$ \\ ' Dermatologikum Hamburg, Hamburg, Deutschland \\ ${ }^{2}$ Klinik für Hautkrankheiten - Allgemeine Dermatologie und Venerologie, Universitätsklinikum Münster \\ Münster, Deutschland
}

\title{
Neue Aspekte zur Histopathologie bei Hautinfektionen
}

In anderen Fällen kann eine Infektion zwar nicht sicher diagnostiziert werden, es findet sich jedoch ein Infiltratmuster („pattern“), das auf eine erregerbedingte Genese hinweist (『Tab. 1; [1, 3]). Für den Histopathologen ist es von größter Wichtigkeit, diese Reaktionsmuster zu kennen, da er den Kliniker auf die zur Bestätigung der Infektion notwendige Zusatzdiagnostik (Kultur, Serologie) aufmerksam machen sollte. Das paraffineingebettete Material kann außerdem durch molekularbiologische Verfahren wie In-situ-Hybridisierung oder PCR gezielt auf bestimmte Erreger untersucht werden. Die molekularbiologische Erregerdiagnostik ist zwar bisher nur nach GOÄ abrechenbar, jedoch ist die Diagnostik in bestimmten Konstellationen (z.B. bei der frühen Borreliose oder auch bei Leishmanien-Infektionen) den serologischen und kulturellen Methoden in Präzision und Praktikabilität überlegen. So sind in den letzten Jahren insbesondere erregerarme Formen diverser Infektionserkrankungen durch Nachweis spezifischer DNA im Gewebe diagnostizierbar geworden [1, 4]. Studien mit molekularbiologischer Erregerdiagnostik aus Biopsiematerial haben auch wesentlich dazu beigetragen, das histomorphologische Spektrum erregerbedingter Hautinfiltrate besser zu charakterisieren

Im Folgenden soll auf Basis einer selektiven Literaturrecherche und der Auswertung von Einzelfällen auf neue oder noch wenig bekannte Reaktionsmuster und auf differenzialdiagnostische Schwierigkeiten aufmerksam gemacht werden. Neben ungewöhnlichen Herpes-simplex- und Varizella-zoster-Infektionen wird die Histopathologie von Coxsackievirus- und Masernexanthem, Borreliose, Syphilis und das Spektrum der kutanen Leishmaniasis dargestellt.

\section{Ungewöhnliche Manifesta- tionen von Herpes-simplex- und Varizella-Zoster- Virusinfektionen}

Herpes-simplex-Viren Typ 1 und Typ 2 (HSV 1 und 2, Humanes Herpesvirus HHV 1 und 2) und das Varizella-ZosterVirus (VZV) verursachen an der Haut intraepidermale Bläschen auf erythematösem Grund. Die gruppierte Anordnung der Läsionen („herpetiform“) und begleitende Missempfindungen (Brennen bzw. Schmerzen) sind meist so typisch, dass eine Biopsie nicht erforderlich ist. Die Primärinfektion kann jedoch auch klinisch inapparent verlaufen. Die Viren persistieren nachfolgend lebenslang in Nervenstrukturen, von wo aus sie bei reduzierter Abwehrlage Rezidive auslösen können (Herpes simplex recidivans bzw. Zoster).

Histologisch verursachen Herpessimplex- und Varizella-Zoster-Viren sehr vergleichbare Veränderungen mit einer Kombination von Ballonierung (intrazellulärem Ödem), Akantholyse und Nekrose der Keratinozyten [1, 3]. Die intraepidermalen Bläschen entstehen durch Ruptur der Keratinozyten (retikulärer Alteration), Massennekrosen und Akantholyse. Keratinozyten mit graublauen Kernen und promi- 


\section{Patterns}

Beschreibung

Epidermal

Subkorneale Blase

Ballonierung und retikuläre

Alteration

Psoriasiforme Dermatitis

Intraepidermale Pustulation

Intraepidermale Keratinozyten-

nekrosen

Adnexiell

Follikulitis

Follikuläre Keratinozyten-Nekrosen

\section{Dermal}

Lymphozytäre Dermatitis, superfiziell und tief

Interstitielle Dermatitis mit Neutrophilen

Dermal und/oder subkutan

Abszess

Suppurative Granulome

Tuberkuloide Granulome

Granulome mit schaumigen Makrophagen

Lymphozytäre nodulär bis diffus („pseudolymphomatös“) Epidermis perifollikulär Follikelepithel Kernstaub ma
Akantholyse im oberen Stratum granulosum mit Abrundung der Zellen, Ablösung der korbgeflechtartig orthokeratotischen Hornschicht

Intraepidermales intrazelluläres Ödem, Ruptur der Keratinozyten mit retikulären Zellresiduen, dann intraund subepidermale Blasenbildung

Akanthose der Epidermis, superfizielle perivaskuläre lymphozytär betonte Infiltrate

Neutrophile intraepidermal aggregiert, variable Spongiose, superfizielle perivaskuläre Infiltrate

Einzeln oder in Clustern liegende Zellnekrosen in der

Dichte Infiltrate von Neutrophilen im Follikel, ggf. auch

Einzeln oder in Clustern liegende Zellnekrosen im

Keine wesentliche epidermale Beteiligung, Infiltrate betont um den superfiziellen und tiefen Gefäßplexus

Keine wesentliche epidermale Beteiligung, perivaskulär gemischte Infiltrate, interstitiell Neutrophile und

Dichte Aggregationen von Neutrophilen in der Dermis/ Subkutis, evtl. Fistelbildung

Makrophagen um zentral aggregierte Neutrophile, umgebend Lymphozytensaum

Aggregationen von Makrophagen mit Lymphozytensaum, evtl. zentrale Nekrose

Makrophagen zeigen ein fein vakuolisiertes Zytoplas-

Dichte Aggregationen von Lymphozyten in der Dermis/ Subkutis mit Beimengung andere Zellen
Mögliche Erreger

Staphylokokken

Herpes-, Orthopox-, Parapoxvieren, Coxsackieviren

Dermatophyten, Candida, Treponema pallidum, Skabies

Staphylokokken, Streptokokken, Dermatophyten, Candida

Herpes, Orthopox-, Parapoxvieren, Coxsackieviren

Staphylokokken/Streptokokken, gramnegative Keime, Pityrosporum, Candida, Dermatophyten, Demodex, Herpes

Masernexanthem, Herpes

Borrelien, Herpes, Virusexantheme

Streptokokken, Erysipelothrix rhusiopathiae

Staphylokokken, Streptokokken, gramnegative Erreger, Nokardien, Aktinomyzeten, Bartonella

Staphylokokken, Streptokokken, gramnegative Erreger, Nokardien, Aktinomyzeten, Bartonella, Dermatophyten, atypische Mykobakterien, Erreger der "tiefen Mykosen"

Mycobacterium tuberculosis, Mycobacterium leprae, Treponema pallidum, Leishmania, Bartonella

Mycobacterium leprae, Klebsiella rhinoscleromatis, E. coli

Borreliose, Leishmaniasis, Skabies, Arthropodenreaktion nenter Kernmembran (marginalisiertes Kernchromatin) und der Nachweis von multinukleären Keratinozyten sind weitgehend diagnostisch, erlauben jedoch keine Unterscheidung zwischen HSVund VZV-Infektion.

Gelegentlich bietet sich jedoch klinisch ein uncharakteristisches Bild mit flächigen Erythemen oder erythematösen Papeln. Dies ist insbesondere für sehr frühe Läsionen und auch für Infektionen mit Beteiligung der Adnexepithelien beschrieben worden (Herpesfollikulitis/ Sycosis herpetica und Herpessyringitis) [5]. In solchen Fällen können die charakteristischen epidermalen Verän- derungen gänzlich fehlen und auch am Adnexepithel finden sich oft keine Akantholyse und kaum Ballonierung. Das Infiltratmuster einer Follikulitis mit basalen und suprabasalen Keratinozytennekrosen oder auch eine vollständige Nekrose des Follikelepithels sind diagnostisch hinweisend (• Abb. 1a, b). Im Follikelepithel können multinukleäre Keratinozyten und graublaue Zellkerne auftreten und sollten gezielt gesucht werden (evtl. Serienschnitte notwendig). Interessanterweise wird eine prominente Follikelbeteiligung insbesondere bei VZV-Reinfektionen (Zoster) gesehen, was mit der ausgeprägten Innervati- on des Follikels und der neurotropen Wanderung der Viren begründet wurde. Im Epithel der ekkrinen Drüsen finden sich bei der Herpes-Syringitis ebenfalls Keratinozytennekrosen sowie ein auffällig periekkrin lokalisiertes Infiltrat, während Akantholyse selten vorliegt.

Der spezifische Nachweis von Herpesviren ist durch Immunhistochemie, Insitu-Hybridisierung und PCR am Biopsiematerial möglich. Dies ist insbesondere dann hilfreich, wenn diagnostische zytopathische Merkmale nicht vorliegen und auch das klinische Bild nicht sicher diagnostisch ist. In manchen Fällen findet sich lediglich ein superfiziel- 
les und tiefes lymphozytäres Infiltrat mit perifollikulärer Beteiligung, etwas vergrößerten Lymphozyten und Erythrozytenextravasaten (• Abb. 1c, d; [5]). Diese als „Herpes incognito“ bezeichnete Variante ist nur durch die oben genannte Zusatzuntersuchungen diagnostizierbar [6]. Erythrozytenextravasate resultieren aus einer Endothelschädigung durch die Herpesviren. In seltenen Fällen kann die Endothelschädigung dominieren und das Bild einer nekrotisierenden Vaskulitis mit Fibrineinlagerungen in Gefäßwänden und Gewebenekrose entstehen (• Abb. 1e,f). Derartige Veränderungen weisen auf einen schweren Verlauf der Herpesvirusinfektion hin, der auch klinisch hämorrhagisch nekrotisierend in Erscheinung tritt und als sog. Herpessepsis lebensbedrohlich verlaufen kann.

Als postherpetische isotope Hautreaktion („Wolf's isotopic response“) bezeichnet man sekundäre Veränderungen einer anderen Hauterkrankung im Areal einer vorangegangenen Herpesvirusinfektion, z.B. bei Psoriasis vulgaris oder Lichen ruber oder auch als postherpetische granulomatöse Dermatitis [7]. Bei Patienten mit hämatologische Grunderkrankung können sich im Bereich der Herpesvirusinfektion spezifische Infiltrate ausbilden, die klinisch einen „nicht abheilenden Herpes“ simulieren [6]. Bei einer nach Therapie nicht abheilenden Herpesvirusinfektion kann daher anhand der Hautbiopsie zwischen einer postherpetischen isotopen Hautreaktion, einem leukämischen Infiltrat und einer tatsächlichen Persistenz der Virusinfektion differenziert werden.

\section{Masernexanthem}

Die Masern gehören zu den ansteckendsten Infektionskrankheiten und können mit Pneumonie und Meningoenzephalitis potenziell tödlich verlaufen [8]. Sie werden durch das Masernvirus hervorgerufen, ein ausschließlich humanpathogenes einzelsträngiges RNA-Virus aus der Familie der Paramyxoviren. Die Masernimpfungen bietet lebenslange Immunität, jedoch bestehen weltweit und auch in Deutschland große Impflücken. Gerade sind laut Weltgesundheitsorganisation (WHO) bei einem Masernausbruch im

Wien klin Mag 2021 · 24:20-33 https://doi.org/10.1007/s00740-020-00379-5

(c) Der/die Autor(en) 2020

\section{A. Böer-Auer \\ Neue Aspekte zur Histopathologie bei Hautinfektionen}

\section{Zusammenfassung}

Hintergrund. Bei entzündlichen Hautinfiltraten ist grundsätzlich immer auch an eine erregerbedingte Genese zu denken. Während einige Erreger bereits in der HämatoxylinEosin-Färbung gut sichtbar sind (z. B.

Hefepilze, Leishmanien), stehen für andere histochemische und immunhistochemische Färbungen zur Verfügung.

Fragestellung. Bei fehlendem Erregernachweis kann eine Infektionserkrankung am Schnittpräparat oft nicht sicher diagnostiziert werden, jedoch kann das Infiltratmuster („pattern“) eine erregerbedingte Genese nahelegen. Es soll auf neue oder noch wenig bekannte Reaktionsmuster und auf differenzialdiagnostische Schwierigkeiten aufmerksam gemacht werden.

Material und Methoden. Selektiver Literaturreview und Auswertung von Einzelfällen.

Ergebnisse. Studien mit molekularbiologischer Erregerdiagnostik aus Biopsiematerial haben wesentlich dazu beigetragen, das histomorphologische Spektrum erregerbedingter Hautinfiltrate besser zu charakterisieren. Neben ungewöhnlichen Herpes-simplex- und Varizella-zosterInfektionen wird die Histopathologie von Coxsackievirus und Masernexanthem, Borreliose, Syphilis sowie der kutanen Leishmaniasis dargestellt. Für zahlreiche Erreger sind inzwischen molekularbiologische Nachweise etabliert, die am formalinfixierten, paraffineingebetteten Material durchgeführt werden können.

Schlussfolgerungen. Anhand von ausgewählten Hautinfektionen wird das breite histopathologischen Spektrum erregerbedingter Hautinfiltrate dargestellt. Für den Histopathologen ist es insbesondere wichtig zu wissen, bei welchen Reaktionsmustern er den Kliniker auf notwendige Zusatzdiagnostik (Kultur, Serologie) aufmerksam machen sollte und wann eine molekularbiologische Erregerdiagnostik aus Biopsiematerial sinnvoll sein kann.

\section{Schlüsselwörter}

Infiltratmuster · Borreliose · Coxsackie . Leishmaniose · Syphilis · Masern

\section{New aspects in the histopathology of infectious skin diseases}

\section{Abstract}

Background. An infectious pathogenesis should always be considered in inflammatory infiltrates in the skin. While some organisms can be recognized on hematoxylin-eosin staining (e.g. yeasts, leishmania), histochemical and immunohistochemical stainings are available for others.

Objectives. If no organisms are seen in a section, the diagnosis of an infection cannot be made with surety, but the pattern of the inflammatory infiltrate can still be suggestive of an infectious process. New or little-known reaction patterns and difficulties in differential diagnosis will be demonstrated. Materials and methods. Selective literature review and analysis of individual cases. Results. Studies using molecular techniques to identify organisms in biopsy specimens have helped to better characterize the histomorphological spectrum of skin infiltrates in infectious skin diseases. Apart from unusual herpes simplex and varicella zoster infections, the histopathology of coxsackie virus and measles exanthem, borreliosis, syphilis, and of cutaneous leishmaniasis is demonstrated. For numerous organisms, molecular tests have been established that can be used on the formalin-fixed, paraffin-embedded material. Conclusions. Selected skin infections demonstrate the broad histomorphological spectrum of skin infiltrates induced by infectious organisms. It is important for histopathologists to know which reaction pattern requires them to alert the clinician to necessary ancillary diagnostics (culture, serology) and when to consider molecular diagnostics to be performed on the biopsy specimen.

\section{Keywords}

Pattern · Borreliosis · Coxsackie · Leishmaniasis · Syphilis · Measles 

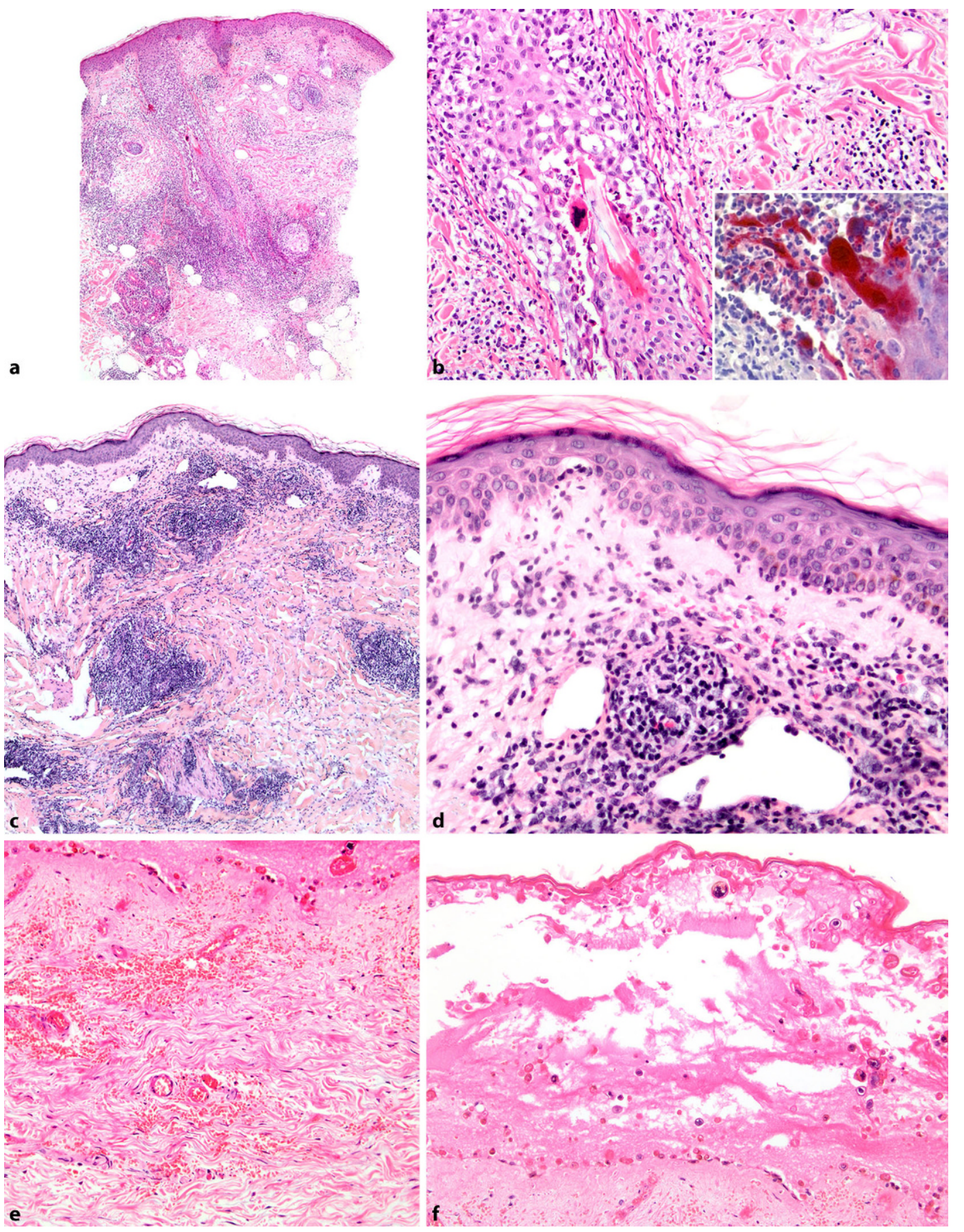

Abb. $1<$ Herpesvirus-Infektionen. $\mathbf{a}$, b Herpesfollikulitis mit Keratinozytennekrosen im Follikelepithel und einer einzelnen mehrkernigen Epithelzelle (b, Insert). c, $\mathbf{d}_{\text {"H }}$ Herpes incognito". Diagnostische epitheliale Veränderungen fehlen. Das Infiltrat ist tiefreichend, lymphozytär mit Erythrozytenextravasaten und einzelnen Lymphozyten in der basalen Epidermis (d).e, f Herpesinfektion mit vaskulitischen Veränderungen (Fibrin in Gefäß wänden, Erythrozytenextravasate). Die nekrotische Epidermis zeigt noch Akantholyse und mehrkernige Keratinozyten (f). Immunhistochemischer Nachweis Varizella-Zoster-Virus-befallener Zellen ( $f$ )

Kongo mehr als 6000 Menschen gestorben. Im südpazifischen Inselstaat Samoa existierte im vergangenen Dezember der Masernnotstand mit mehr als 5100 infizierten und 72 verstorbenen Patienten. In Deutschland wurden bis Mitte Oktober 2019 bereits 501 Fälle registriert [8]. Während sich im Kindesalter oft ein klinisch typisches Bild mit zunächst katarrhalischen Symptomen und nachfolgendem makulopapulösen Exanthem mit Gesichts- und Schleimhautbeteiligung zeigt, können bei Erwachsen uncharakteristische Beschwerden vorliegen. Die Abgrenzung des Exanthems von Arzneimittelexanthemen oder anderen Virusexanthemen ist klinisch nicht immer möglich oder es wird an die Diagnose der Masern gar nicht gedacht. In solchen Fällen kann die Entscheidung für die Hautbiopsie fallen. Histopathologisch sieht man in der Dermis ein mildes perivaskuläres superfizielles und tiefes Infiltrat aus Lymphozyten und Histiozyten mit einzelnen Neutrophilen und Eosinophilen (• Abb. 2a-f). Während die epidermalen Veränderungen mit etwas Vakuolisierung und Spongiose an eine Arzneireaktion denken lassen können, finden sich im Follikelepithel und in Talgdrüsenepithelien nekrotische Keratinozyten (einzeln und in kleinen Clustern) und multinukleäre Zellen, 

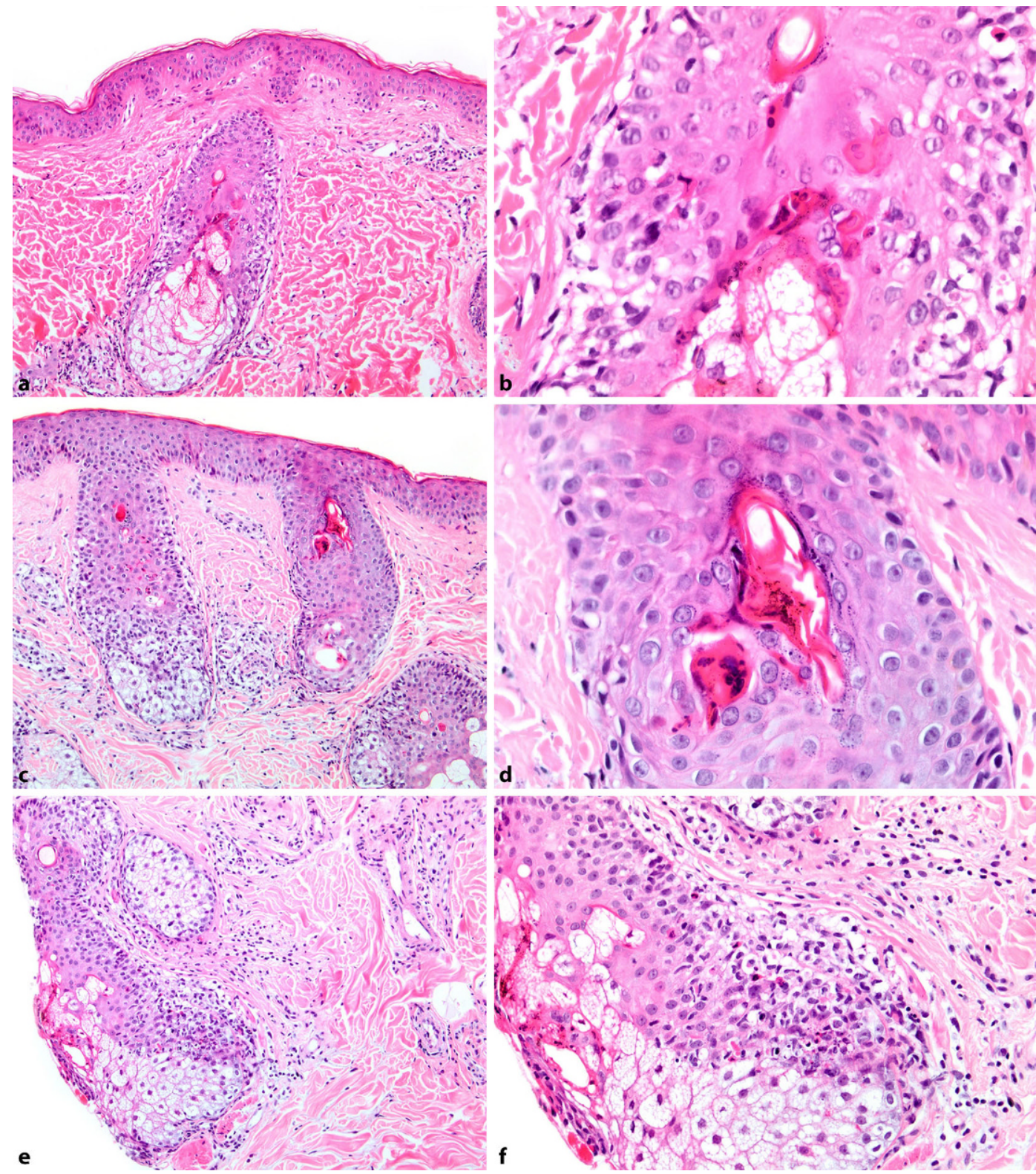

Abb. $2 \triangleleft$ Masernexanthem. a, b Nekrotische Keratinozyten im Infundibulum. c, d Schüttere lymphozytäre Infiltrate und eine multinukleäre Zelle im Infundibulum. e, $\mathrm{f}$ Zellnekrosen im Talgdrüsenepithel

jedoch keine Ballonierung oder Akantholyse. Vereinzelt finden sich synzytiale Riesenzellen auch in der Epidermis [9, 10]. Die Follikelbeteiligung des Exanthems ist jedoch hochcharakteristisch und muss nötigenfalls anhand von Serienschnitten gesucht werden. Hilfreich ist der klinische Hinweis auf eine Gesichtsbeteiligung des Exanthems. Eine Biopsie aus gesichtsnaher, follikelreicher Haut bietet eine bessere Chance, die typischen Nekrosen im Follikel- und Talgdrüsenepithel aufzufinden. Zusätzlich kann die immunohistochemische Färbung mit Anti-Measles-Virus(MeV)-
Nucleoprotein und Anti-MeV-Phosphoprotein diagnostisch hilfreich sein [10].

\section{Coxsackievirusexanthem (Hand- Fuß-Mund-Krankheit)}

Coxsackieviren sind unbehüllte Einzel(+)-Strang-RNA-Viren (Gattung Enterovirus, Familie Picornaviridae) Die Coxsackieviren rufen vor allem Erkältungssymptome, aber auch die virale Meningitis und Myokarditis hervor. An der Haut verursachen Coxsackie-AViren (A2-A8, A10, A12, A14, A16) und Enterovirus A71 (EV-A71) die Hand-Fuß-Mund-Krankheit, eine meist im Kindesalter $(<10$ Jahre) auftretende Erkrankung mit Fieber und Bläschen sowie Ulcera im Mund sowie einem makulopapulösen Exanthem an Händen und Füßen und komplikationslosem Verlauf [11]. In der aktuellen Literatur finden sich jedoch zunehmend Berichte über Hand-Fuß-Mund-Krankheit bei Erwachsenen, oft mit ungewöhnlichem klinischen Bild mit Beteiligung von Gesicht und Stamm und Ähnlichkeiten zum Erythema exsudativum multiforme (EEM) oder schweren Arzneireaktionen $[12,13]$. Es wurde auch eine häufige genetische Modifikation der Virusstämme beobachtet, teilweise mit Änderung des 
Hier steht eine Anzeige.

\section{曾 Springer}



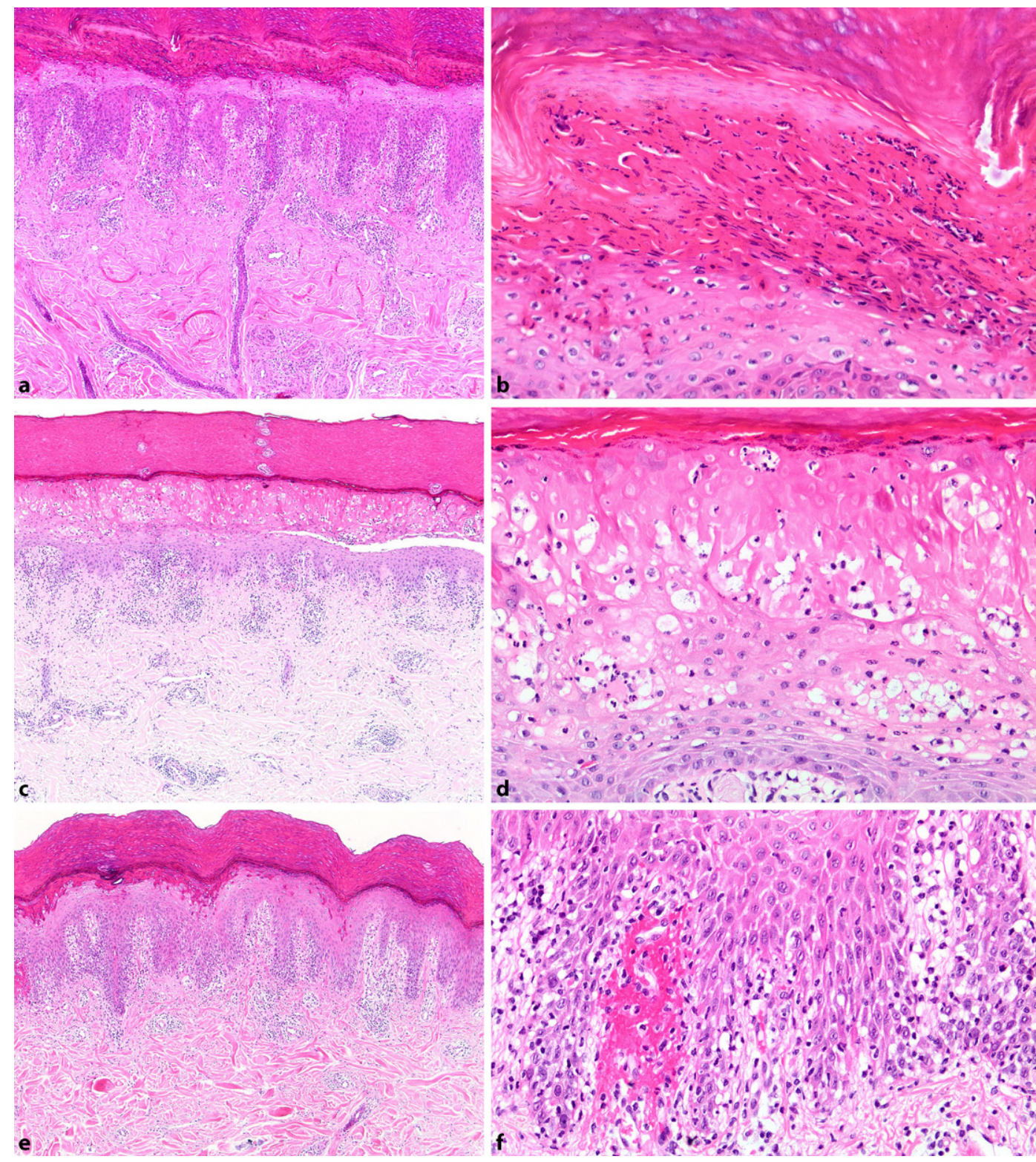

Abb. $3 \triangleleft$ Hand-FußMund-Krankheit (Coxsackievirusinfektion). a, b Nekrose des oberen Epidermisdrittels durchsetzt von Neutrophilen $c$, d Ballonierung und retikuläre Alteration des oberen Epidermisdrittels mit Neutrophilen. e, f Nekrose der oberen Epidermis und Infiltrate aus Lymphozyten und Neutrophilen sowie Erythrozytenextravasate in der basalen Epidermis und papillären Dermis

klinischen Bildes mit neurologischen oder kardiologischen Komplikationen.

Erst unlängst wurde die Histopathologie der Hand-Fuß-Mund-Krankheit bei Erwachsenen genauer untersucht (• Abb. 3a-f; [14]). Es zeigt sich eine Kombination aus Spongiose, Ballonierung und retikulärer Alteration kombiniert mit Keratinozytennekrosen in höheren Epidermisschichten. Zusätzlich finden sich teils dicht aggregierte neutrophile Granulozyten subkorneal oder im Verlauf intrakorneal. Im dermalen Infiltrat dominieren Lymphozyten, jedoch sind Neutrophile immer vorhanden und meist auch zahlreich. Erythrozytenextravasate bestehen oft in der papillären
Dermis und Epidermis. Der Neutrophilenreichtum und die präferenzielle Lokalisation der Keratinozytennekrosen in oberen Epithelschichten erlauben in der Regel die Abgrenzung des Hand-FußMund-Exanthems vom EEM. Bei voll entwickelten Läsionen mit vollständiger Epidermisnekrose kann die Abgrenzung beider Erkrankungen jedoch schwierig sein. Die immunhistochemische Färbung auf Enteroviren ist an Biopsien der Hand-Fuß-Mund-Krankheit nicht verlässlich [15]. Es wurde vermutet, dass das Exanthem parainfektiös entsteht und der Erreger in der Haut möglicherweise gar nicht vorliegt, jedoch gelang kürzlich der Virusnachweis aus Blasenflüssigkeit mittels Reverse-Transkription-PCR [15].

\section{Borreliose}

Die Borreliose ist die häufigste durch Zecken übertragene Infektionskrankheit in Europa [16]. Borrelien sind relativ große, schraubenförmige/spiralförmige, gramnegative Bakterien (Spirochäten). Sie gelangen über den Stich der Schildzecke Ixodes ricinus in die Haut und verursachen das Erythema (chronicum) migrans oder ein Borrelien-Lymphozytom. Im Verlauf kann es zur disseminierten und chronischen Infektion kommen, bei der neben der Haut (Acrodermatitis 


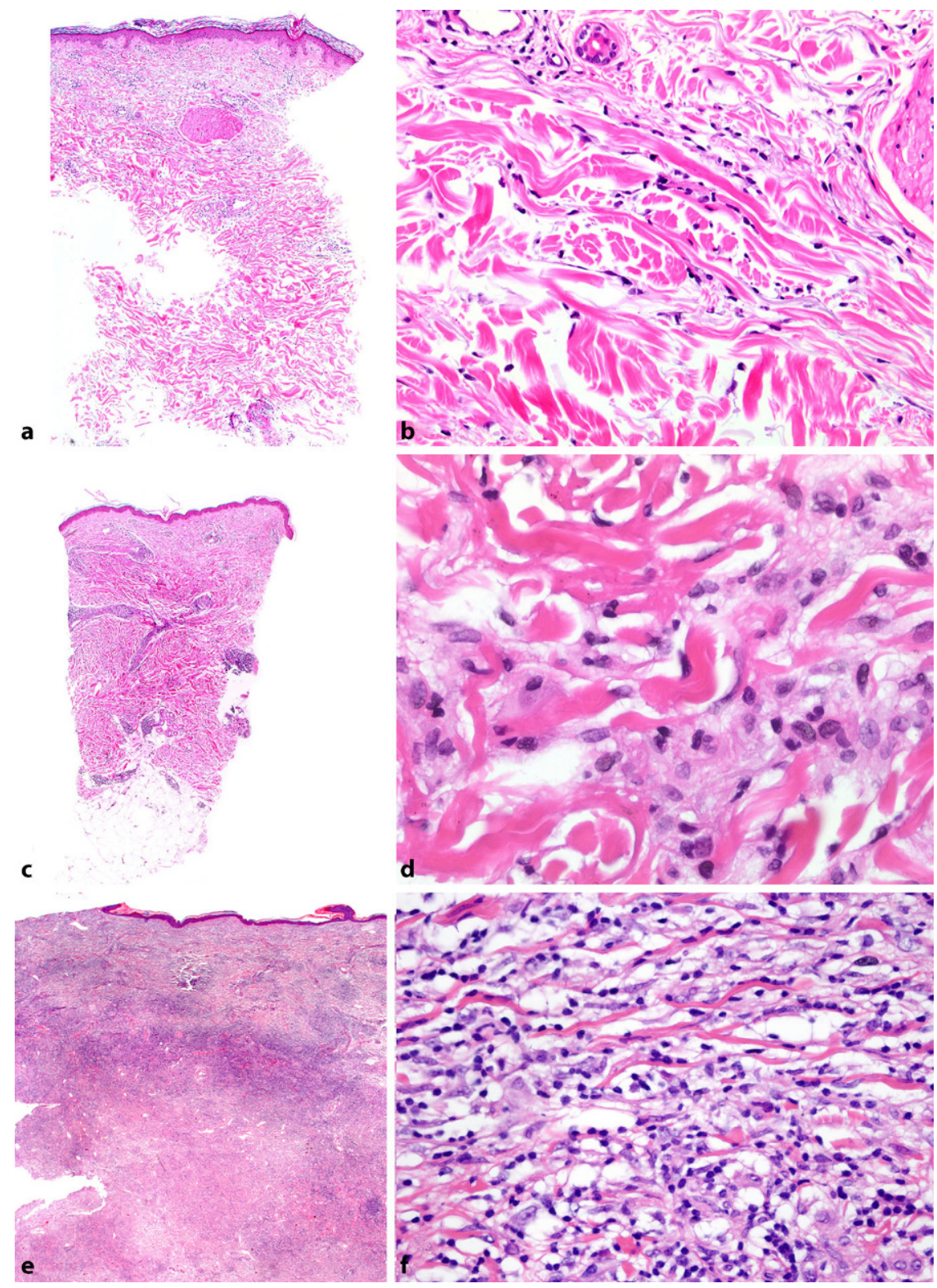

Abb. 4 A Kutane Borreliose. a, b Spärliches lymphozytäres Infiltrat perivaskulär und interstitiell, superfiziell und tief bei multiplen Erythemata migrantia. c, d Perivaskuläre lymphozytäre Infiltrate mit interstitiellen granulomatösen Infiltraten bei disseminierter chronischer Borreliose. e, f Diffuse plasmazellreiche Infiltrate mit interstitiellen Granulomen bei juxtaartikulären Knoten bei chronischer kutaner Borreliose

chronica atrophicans) auch Gelenke, Nervensystem (sog. Neuroborreliose) und Herz betroffen sein können. Die Vektor-Pathogen-Interaktion ist sehr komplex und dauert mehr als $10 \mathrm{~h}$, da die Borrelien erst im Darm der Zecke aktiviert werden, dann zur Speicheldrüse der Zecke wandern und dort an Speichelproteine binden, mit denen sie bei Biss in die menschliche Haut gelangen. Die Diagnose der Borreliose erfolgt in der Regel anhand des klinischen Be- die angeborene und erworbene Immunabwehr unterlaufen („immune escape“) [18]. Serologische Untersuchungen sind insbesondere bei Frühinfektionen wenig verlässlich, zumal Reinfektionen bei Risikopatienten häufig sind [16]. Beweisend ist die Anzucht des Erregers aus Biopsiematerial, jedoch ist die Kultur von Borrelien immer noch äußerst schwierig und nur wenigen Speziallabors vorbehalten [16]. Bei klinisch untypischem Befund wird daher nicht selten biopsiert. Inzwischen liegen einige Studien zur Histopathologie der kutanen Borreliose mit molekularbiologischer Korrelation vor. Dabei hat sich das histomorphologische Spektrum als deutlich breiter erwiesen als zuvor bekannt [19, 20].

Beim Erythema migrans zeigt sich ein superfizielles und konsistent tiefes perivaskuläres und interstitielles Infiltrat aus Lymphozyten ohne wesentliche epidermale Beteiligung (• Abb.4a, b). Einzelne intrabasale Lymphozyten können vorliegen. Die dermale Infiltratdichte ist sehr variabel und mitunter sehr spärlich. Es wurden sowohl T-Zell- als auch B-Zell-dominierte Infiltrate beschrieben. Plasmazellen gelten als typisch, werden jedoch nicht immer angetroffen. Eine makrophagenreiche Variante mit Ähnlichkeiten zum interstitiellen Granuloma anulare wurde beschrieben (- Abb. 4c, d; $[19,20])$. Der Nachweis von borrelienspezifischer DNA im Biopsiematerial erlaubt eine zweifelsfreie Diagnose und ermöglicht zudem die Identifikation der Borrelienspezies, kann jedoch bei geringer Erregerdichte auch negativ sein [19, 20]. Beim Borrelienlymphozytom bestehen knotige Infiltrate, die klinisch und histologisch ein Lymphom imitieren können. Infiltrate sind gemischt und bestehen aus B- und T-Lymphozyten, Makrophagen sowie auch aus Eosinophilen und Plasmazellen. Reaktive Keimzentren können sich ausbilden. Das klinische Bild kann wegweisend sein, da Borrelien-Lymphozytome sich gerne an Ohrläppchen oder Brustwarzen manifestieren. Molekularbiologisch sind die Infiltrate polyklonal. Da pseudolymphomatöse Infiltrate auch durch andere Auslöser (z.B. Insektenstich, Fremdmaterial etc.) entstehen können, ist ein Nachweis von borrelienspezifi- 
a
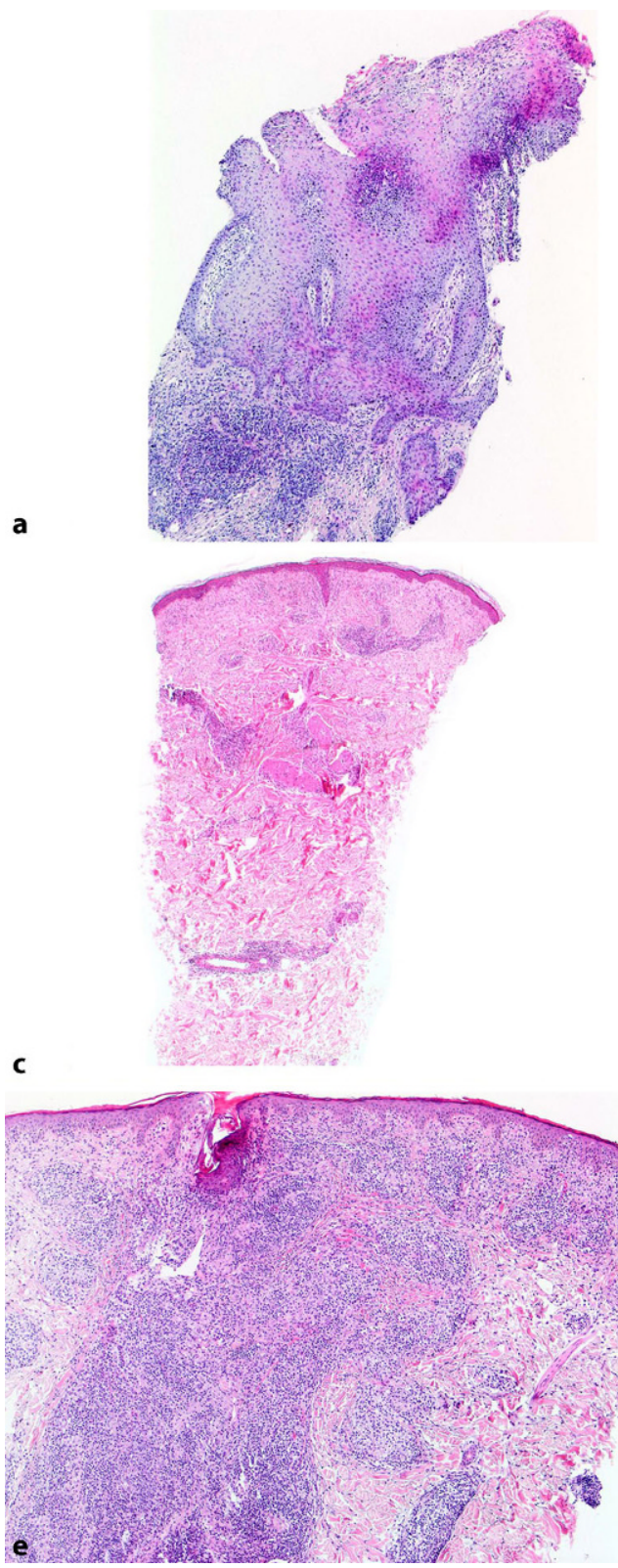

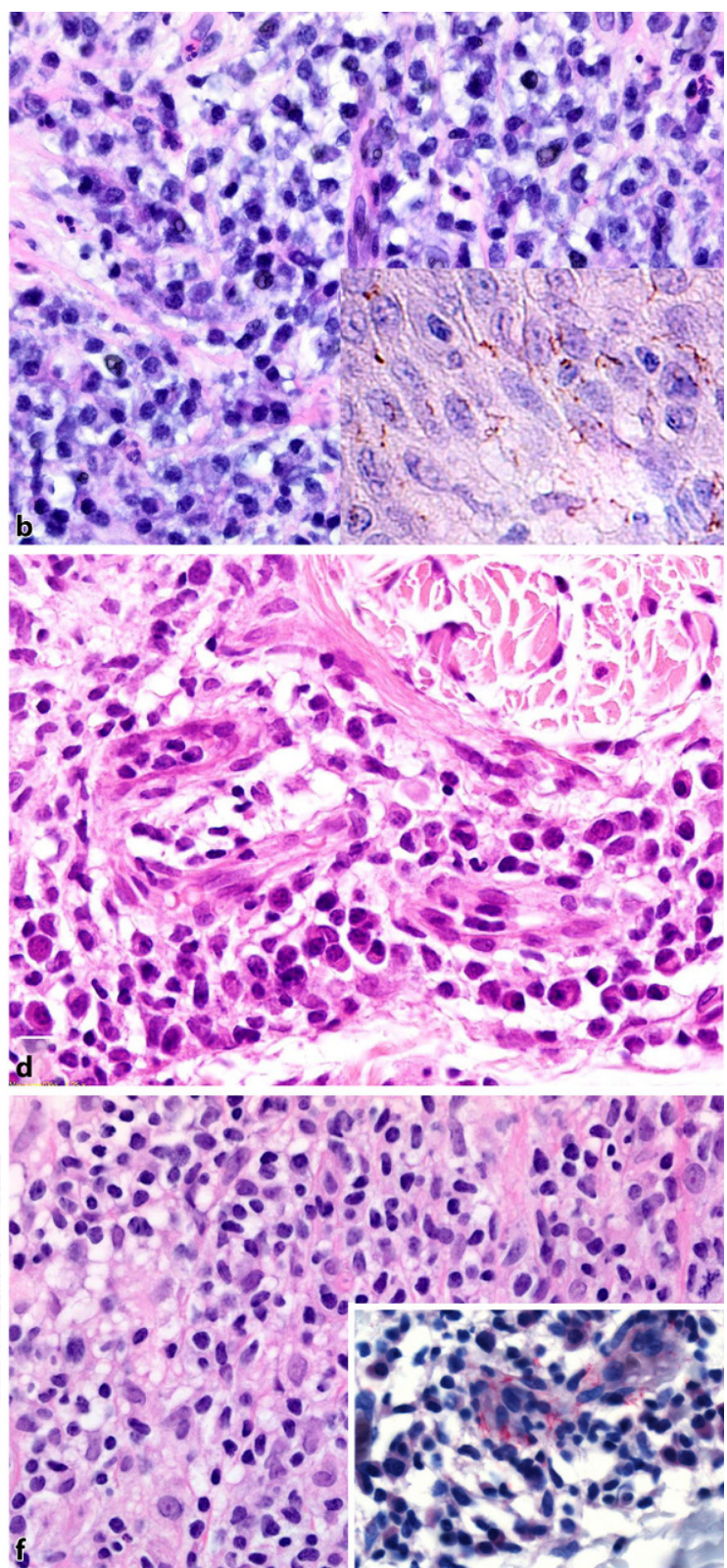

Abb. $5<$ Lues. a, b Primäraffekt mit ausgeprägter epithelialer Hyperplasie und dichtem Plasmazellinfiltrat; immunhistochemischer Nachweis von Treponema pallidum intraepithelial (b, Insert). c, d Sekundäre Syphilis mit plasmazellreichem Infiltrat, jedoch atropher Epidermis. e, f Tertiäre Syphilis mit beginnender Granulombildung; immunhistochemischer Nachweis von Treponema pallidum in Makrophagen (f, Insert) scher DNA im Biopsiematerial oder eine serologische Bestätigung der Infektion zur sicheren Diagnose notwendig. Die kutane Spätmanifestation der Borreliose, die Acrodermatitis chronica atrophicans ist klinisch sowie auch histologisch distinkt [20]. Die meist unilateralen, extremitätenbetonten, lividen, atrophen Erytheme zeigen subepidermal bandförmige bis diffuse Infiltrate von Lymphozyten, Makrophagen und Plasmazellen begleitet von epidermaler Atrophie und sklerotischem Kollagen. Der Nachweis von borrelienspezifischer DNA im Biopsiematerial erlaubt eine zweifelsfreie Diagnose, jedoch ist die Acroderma- titis chronica atrophicans nicht selten erregerarm, sodass die PCR-Diagnostik auch negativ sein kann [20]. Auch bei der chronischen Borreliose sind klinische Varianten beschrieben worden, so z. B. disseminierte Erytheme und fibroide juxtaartikuläre Knoten (-Abb. 4e, f) und eine septolobuläre Pannikulitis [21]. In einigen Fällen finden sich prominente interstitielle Granulome sowie auch Muzineinlagerungen mit Ähnlichkeiten zum Granuloma anulare [19, 20]. Bei der chronischen Borreliose ist jedoch die Borrelien-Serologie in der Regel hochpathologisch, sodass diese zur Bestätigung herangezogen werden kann [16].

\section{Syphilis (Lues)}

Die Syphilis ist eine sexuell übertragbare Infektion durch die gramnegative Spirochäte Treponema pallidum. Die Erkrankung ist weltweit verbreitet mit ca. 12 Mio. Fällen pro Jahr zu über $90 \%$ in Entwicklungsländern [22, 23]. In Deutschland sind vor allem Großstädte betroffen und hier zumeist homosexuelle Männer mit 3-6 Neuinfektionen je 100.000 Einwohnern pro Jahr. Oft besteht eine Koinfektion mit HIV [24]. Die Syphilis zeigt einen stadienhaften Verlauf: primär (Lues I), Ulcus durum, regionale Lymphadenopathie; sekundär 

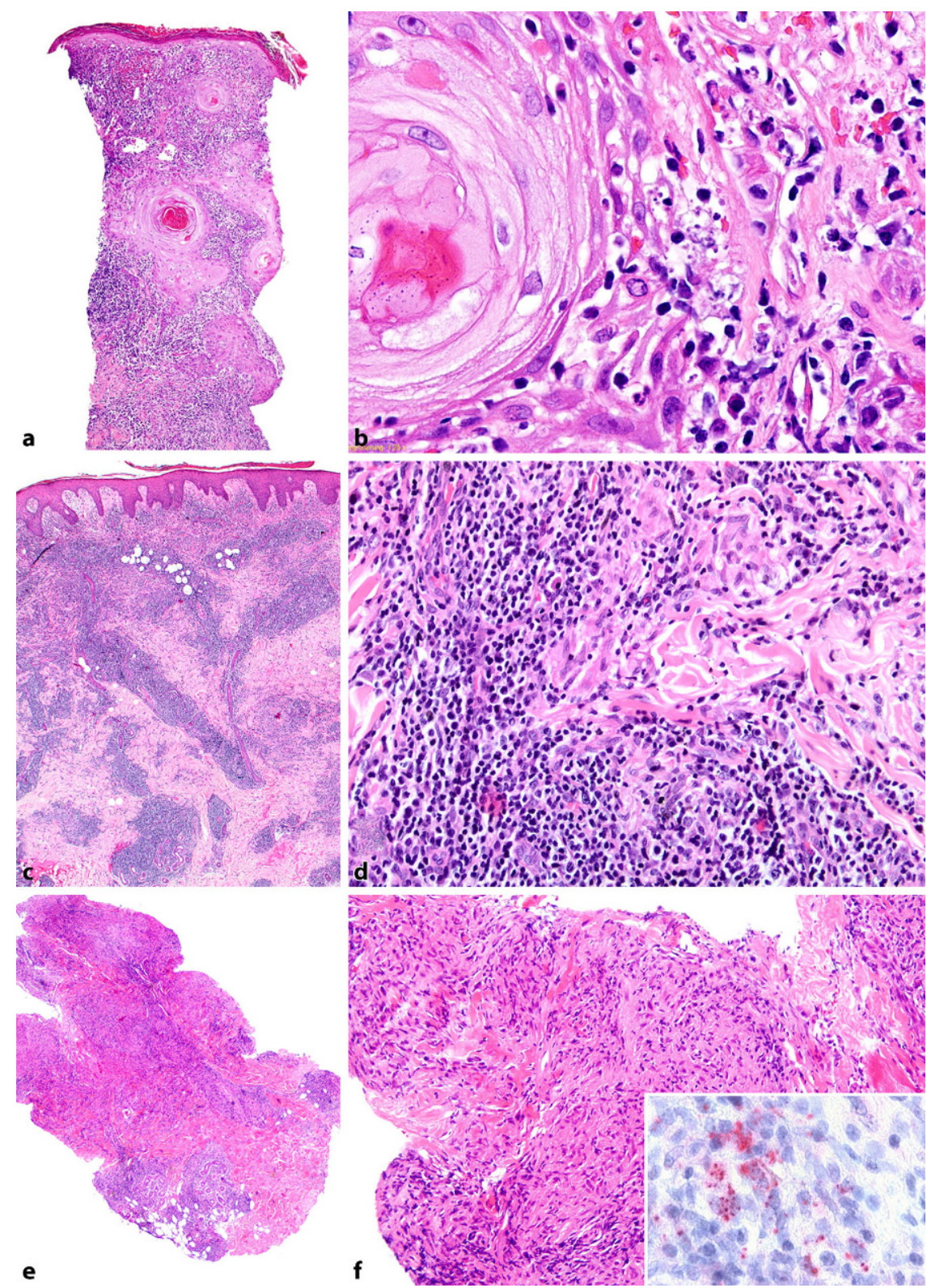

Abb. $6 \Delta$ Kutane Leishmaniose. a, b Ausgeprägte pseudokarzinomatöse Hyperplasie bei Infektion mit L. panamnensis.c, d Pseudolymphomatöse Infiltrate, nur sehr fokale Granulome bei L. major. e, f Sarkoidale Granulome bei Infektion mit L. kilicki. Immunhistochemischer Nachweis von Leishmanien (f, Insert)

(Lues II), stammbetontes Exanthem, evtl. Condylomata lata, fleckförmige Alopezie (Alopecia specifica); tertiär (Lues III), Gummen, evtl. Multiorganbefall Herz, große Gefäße, Skelett und Nervensystem; Neurolues (Lues IV) als Spätkomplikation nach jahrzehntelangem Verlauf mit Demenz, Ataxie, Wahrnehmungsstörungen, Tabes dorsalis [1]. Bei klinischem Verdacht auf eine Syphilis ist eine serologische Diagnostik zur Bestätigung zu veranlassen. Am Primäraffekt kann nom imitieren (- Abb. 5a, b; [1, 3]). Für die sekundäre Syphilis wurden psoriasiforme Hyperplasie der Epidermis kombiniert mit bandförmigen subepidermalen Infiltraten und am Kapillitium perifollikulären Infiltraten beschrieben. Die Follikelbeteiligung führt zum klinischen Bild der Alopecia specifica (mottenfraßähnlicher Haarausfall). In manchen Fällen fehlt jedoch die Epidermishyperplasie (•Abb. 5c, d). Typisch sind vergleichsweise dichte Infiltrate mit zahlreichen Plasmazellen und Makrophagen im Infiltrat [25], es wurden jedoch auch plasmazellarme Manifestationen beschrieben. Findet sich zusätzlich eine nekrotisierende Vaskulitis, so kann dies auf eine sog. Lues maligna mit klinisch ulzerierenden Läsionen und schwerem allgemeinen Krankheitszustand hinweisen. Meist sind die Patienten immunsupprimiert. Bei der tertiären Syphilis zeigen sich Granulome (• Abb. 5e, f), die bei der Ausbildung von Gummen auch ausgedehnte Nekrosezonen gesäumt von gemischten Infiltraten mit Plasmazellen, Lymphozyten, Makrophagen und Riesenzellen vom Langhans-Typ sowie Gefäßproliferate zeigen können.

Treponema pallidum kann im Gewebe mit Versilberungstechnik nach WarthinStarry (Cave unspezifische Silberniederschläge), durch spezifische immunhistochemische Antikörper oder mittels PCR nachgewiesen werden [26]. In späten Stadien der Syphilis sind häufig nur sehr wenige Erreger im Gewebe vorhanden, sodass eine negative PCR die Diagnose nicht sicher ausschließt. Eine serologische Diagnostik muss dennoch immer erfolgen.

\section{Kutane Leishmaniasis}

Die kutane Leishmaniasis wird durch Protozoen des Genus Leishmania verursacht. Leishmanien finden sich insbesondere im Mittelmeerraum, im Nahen und Mittleren Osten, Asien und Afrika (kutane Leishmaniasis der Alten Welt) sowie auch in Mittel- und Südamerika (kutane Leishmaniasis der Neuen Welt). In Deutschland tritt die Erkrankung typischerweise als importierte Infektion nach Reisen in den Mittelmeerraum auf [1]. Die Übertragung erfolgt durch Sand- 
mücken (Phlebotomus, Lutzomyia und Psychodopygus), in denen die unbewegliche amastigote Form zur infektiösen begeißelten Promastigote heranreift. Die kutane Leishmaniasis wird aufgrund der ausschließlichen Hautmanifestation von der mukokutanen Leishmaniasis und der viszeralen Leishmaniasis (Kala-Azar) abgegrenzt. Wochen bis Monate nach dem Stichereignis entsteht typischerweise auf unbedeckten Hautarealen eine erythematöse Papel, die zu einem Knoten oder Plaque heranwächst und im Verlauf ulzeriert. Mitunter finden sich multiple Läsionen, manchmal auch in sporotrichoider Ausbreitung. Sofern sich aus der Anamnese kein Hinweis ergibt, besteht klinisch die Schwierigkeit darin, an die Leishmaniose überhaupt zu denken. Das klinische Bild wird je nach Alter des Patienten mit Furunkel, Prurigoknoten, rupturierter Zyste, epithelialen Neoplasien oder auch Lymphomen verwechselt. Daher wird oft eine Biopsie zur differenzialdiagnostischen Abklärung entnommen [1, 27]. Grundsätzlich verursachen Leishmanien eine granulomatöse Gewebereaktion zumeist vom tuberkuloiden Typ mit beigemengten Plasmazellen. Studien mit molekularbiologischem Erregernachweis haben jedoch gezeigt, dass auch andere Granulomtypen vorliegen können, z. B. sarkoidale Granulome, Palisadengranulome oder interstitielle Granulome, sodass sich histologisch jeweils andere Differenzialdiagnosen ergeben (• Abb. 6; [28, 29]). Es sollte bei jeder granulomatösen Dermatitis, die sich klinisch als Einzelläsion auf unbedeckter Haut bietet, an die Möglichkeit der Leishmaniase gedacht werden. Darüber hinaus finden sich jedoch auch leishmanieninduzierte Infiltrate mit lymphozytenreichem Bild in Form von pseudolymphomatösen Infiltraten ( Abb. $6 c, d$ ). Diese finden sich insbesondere bei Infektionen mit L. major [28]. Eine diagnostische Falle sind Leishmanieninfektionen mit ausgeprägter pseudokarzinomatöser Epithelhyperplasie, die ein hochdifferenziertes Plattenepithelkarzinom imitieren können (• Abb. 6a, b). Dies wurde vor allem bei Infektionen mit $L$. major und $L$. panamnensis beobachtet, kann aber auch bei Infektionen mit $L$. infantum vorkommen
[28]. In diesen Fällen kann nur die Identifikation der Amastigoten im Gewebe die Fehldiagnose verhindern. Die Leishmanienamastigoten können bereits in der HE-Färbung als basophile, unbekapselte, ca. $2-4 \mu \mathrm{m}$ große Strukturen erkannt werden, jedoch fällt die sichere Identifikation bei geringer Erregerdichte mitunter schwer. Die Giemsa-Färbung und auch die immunhistochemische Färbung mit CD1a-Antikörper 235 (Kreuzreaktivität mit Leishmanien) der Firma Leica (Leica Biosystems Nussloch $\mathrm{GmbH}$, Nußloch, Deutschland) können bei der Identifikation der Leishmanien helfen. Die Erregerdichte ist oberflächennah im Zentrum der Läsion am höchsten.

Bei Verdacht auf eine kutane Leishmaniasis und fehlendem oder unsicherem Erregernachweis in der Biopsie kann eine molekularbiologische Diagnostik am bereits entnommenen Biopsiematerial durchgeführt werden $[28,29]$. Der Nukleinsäurenachweis ist die sensitivste Methode für den Nachweis von Leishmanien und sie erlaubt zudem auch die exakte Bestimmung der Spezies, was therapeutische Konsequenzen hat, da sich die Leishmanien-Subspezies hinsichtlich ihrer Pathogenität und ihres therapeutischen Ansprechens unterscheiden. Eine serologische Diagnostik ist bei Patienten mit wenigen Läsionen einer kutanen Leishmaniase meist negativ und daher diagnostisch nicht hilfreich. Bei multiläsionalen Manifestationen können hingegen Anti-Leishmania-Antikörper nachweisbar sein.

\section{Fazit für die Praxis}

- Die molekularbiologische Erregerdiagnostik aus Biopsiematerial hat zu eine Präzisierung des histomorphologischen Spektrums erregerbedingter Hautinfiltrate beigetragen.

- Die Identifikation charakteristischer Infiltratmuster (Patterns) ermöglicht einen gezielten Einsatz von Zusatzdiagnostik am Biopsiematerial (Histochemie, Immunhistochemie, Molekularbiologie) oder auch serologischer und kultureller Verfahren zur Bestätigung der Diagnose.

- Die hier dargestellten histopathologischen Merkmale von ungewöhnli- chen Herpes-simplex- und Varizellazoster-Infektionen, der Histopathologie von Coxsackievirus- und Masernexanthem, der Borreliose, der Syphilis sowie der kutanen Leishmaniasis sollen im Histopathologiealltag dafür sensibilisieren, dass sich auch altbekannte Infektionskrankheiten mit neuen Aspekten zeigen können.

- Für zahlreiche Erreger sind inzwischen molekularbiologische Nachweise etabliert, die am formalinfixierten, paraffineingebetteten Material durchgeführt werden können, was vor allem bei erregerarmen Formen hilfreich ist.

- Die molekularbiologische Erregerdiagnostik am Paraffinmaterial ist jedoch bisher nur nach GOÄ abrechenbar.

\section{Korrespondenzadresse}

Prof. Dr. Almut Böer-Auer

Klinik für Hautkrankheiten - Allgemeine

Dermatologie und Venerologie, Universitätsklinikum Münster

Von-Esmarch-Straße 58, 48149 Münster,

Deutschland

boer@dermatologikum.de

Funding. Open Access funding enabled and organized by Projekt DEAL.

\section{Einhaltung ethischer Richtlinien}

Interessenkonflikt. A. Böer-Auer gibt an, dass kein Interessenkonflikt besteht.

Für diesen Beitrag wurden von den Autoren keine Studien an Menschen oder Tieren durchgeführt. Für die aufgeführten Studien gelten die jeweils dort angegebenen ethischen Richtlinien.

Open Access. Dieser Artikel wird unter der Creative Commons Namensnennung 4.0 International Lizenz veröffentlicht, welche die Nutzung, Vervielfältigung, Bearbeitung, Verbreitung und Wiedergabe in jeglichem Medium und Format erlaubt, sofern Sie den/die ursprünglichen Autor(en) und die Quelle ordnungsgemäß nennen, einen Link zur Creative Commons Lizenz beifügen und angeben, ob Änderungen vorgenommen wurden.

Die in diesem Artikel enthaltenen Bilder und sonstiges Drittmaterial unterliegen ebenfalls der genannten Creative Commons Lizenz, sofern sich aus der Abbildungslegende nichts anderes ergibt. Sofern das betreffende Material nicht unter der genannten Creative Commons Lizenz steht und die betreffende Handlung nicht nach gesetzlichen Vorschriften erlaubt ist, ist für die oben aufgeführten Weiterverwendungen des Ma- 
Hier steht eine Anzeige.

\section{曾 Springer}


Pathologie

terials die Einwilligung des jeweiligen Rechteinhabers einzuholen.

Weitere Details zur Lizenz entnehmen Sie bitte der Lizenzinformation auf http://creativecommons.org/ licenses/by/4.0/deed.de.

\section{Literatur}

1. Böer-Auer A (2016) Infektionen der Haut. In: Kerl H, Cerroni L, Garbe C, Kutzner H, Metze D (Hrsg) Histopathologie der Haut. Springer, Berlin, Heidelberg

2. Böer-Auer A (2018) Stellenwert der Biopsie bei der Diagnostik von Infektionserkrankungen der Haut. Hautarzt 69(7):550-562

3. Ackerman AB, Böer A, Benin B et al (2005) Histologic diagnosis of inflammatory skin diseases. An algorithmic method based on pattern analysis, 3. Aufl. Ardor Scribendi, Ltd, New York City. ISBN 1-893357-25-2

4. Sunderkötter C, Becker K, Kutzner H et al (2018) Molekulare Diagnostik von Hautinfektionen am Paraffinmaterial - Übersicht und interdisziplinärer Konsensus. JDtsch Dermatol Ges 16(2):139-148

5. Böer A, Herder N, Winter K et al (2006) Observations clinical, histopathological, and molecular pathologic in herpes folliculitis. Br J Dermatol 154(4):743-746

6. Böer A, Herder N, Blödorn-Schlicht N et al (2006) Herpes incognito, most commonly, is herpes zoster-and its pattern histopathologic is distinctive! Am J Dermatopathol 28(2):181-186

7. Wolf R, Brenner S, Ruocco V, Filioli FG (1995) Isotopic response. Int J Dermatol 34(5):341-348

8. Kouros B, Nennstiel U, Liebl B et al (2019) National immunization program initiatives-what are their contribution to the elimination of measles and rubella in Germany? Bundesgesundheitsblatt Gesundheitsforschung Gesundheitsschutz 62(4):386-391

9. Tirado M, Böer-Auer A (2015) Follicular necrotic keratinocytes-a helpful clue to the diagnosis of measles. JCutan Pathol 42(9):632-638
10. Liersch J, Omaj R, Schaller J (2019) Histopathological and immunohistochemical characteristics of measles exanthema: a study of a series of 13 adult cases and review of the literature. Am J Dermatopathol 41(12):914-923

11. Esposito S, Principi N (2018) Hand, foot and mouth disease: current knowledge on clinical manifestations, epidemiology, aetiology and prevention. Eur J Clin Microbiol Infect Dis 37:391-398

12. Neri I, Chessa MA, Virdi A et al (2018) Hand, foot and mouth disease in adult patients. J Eur Acad Dermatol Venereol 32:e59-e60

13. Balestri R, Bellino M, Landini L et al (2018) Atypical presentation of entero-virus infection in adults: outbreak of "hand, foot, mouth and scalp disease" in northern Italy. J Eur Acad Dermatol Venereol 32:e60-e61

14. Böer-Auer A, Metze D (2019) Histopathology of hand-foot-mouth disease in adults and criteria for differentiation from erythema multiforme. Am J Dermatopathol 41(4):273-280

15. Herrero M, Kutzner H, Fraga J et al (2019) Immunohistochemical study of 2 cases of coxsackie A6induced atypical hand-foot-and-mouth disease. Am JDermatopathol 41(10):741-743

16. Hofmann $\mathrm{H}$, Fingerle V, Hunfeld KP, Consensus group et al (2017) Cutaneous lyme borreliosis: guideline of the German dermatology society. Ger Med Sci 15:Doc14

17. Llamas-Velasco M, Paredes BE (2018) Borrelia lymphocytoma mimicking butterfly rash in a pediatric patient. Am J Dermatopathol 40(3):216-218

18. Tracy KE, Baumgarth N (2017) Borrelia burgdorferi manipulates innate and adaptive immunity to establish persistence in rodent reservoir hosts. Front Immunol 20(8):116

19. Brandt FC, Ertas B, Falk TM et al (2014) Genotyping of borrelia from formalin-fixed paraffin-embedded skin biopsies of cutaneous borreliosis and tick bite reactions by assays targeting the IGS-region, ospA, and OspC genes. Br J Dermatol 171(3):528-543. https://doi.org/10.1111/bjd.12855

20. Brandt CF, Ertas B, Falk TM et al (2015) Histopathology and immunophenotype of acrodermatitis chronica atrophicans correlated with OspA and
OspC genotypes of borrelia species. J Cutan Pathol 42(10):674-692

21. Dittmer MR, Willis MS, Selby JC et al (2018) Septolobular panniculitis in disseminated lyme borreliosis. J Cutan Pathol 45(4):274-277

22. Angus J, Langan SM, Stanway A et al (2006) The many faces of secondary syphilis. Clin Exp Dermatol 31:741-745

23. Gross G, Flaig B, Rode S (2013) Syphilis. Part 1: introduction, pathology and clinical aspects. Hautarzt 64(10):771-788

24. Barreiro $P$ (2018) Hot news: sexually transmitted infections on the rise in PrEP users. AIDS Rev 20(1):71

25. Rysgaard C, Alexander E, Swick BL (2014) Nodular secondary syphilis with associated granulomatous inflammation: case report and literature review. JCutan Pathol 41(4):370-379

26. Behrhof W, Springer E, Bräuninger W et al (2008) PCR testing for treponema pallidum in paraffinembedded skin biopsy specimens: test design and impact on the diagnosis of syphilis. J Clin Pathol 61:390-395

27. Thilakarathne IK, Ratnayake $P$, Vithanage $A$ et al (2019) Role of histopathology in the diagnosis of cutaneous leishmaniasis: a case-control study in Sri Lanka. Am JDermatopathol 41(8):566-570

28. Gebhardt M, Ertas B, Falk TM et al (2015) Fast, sensitive, and specific diagnosis of infections with leishmania species by cytochrome $b$ PCR in formalin-fixed, paraffin-embedded skin biopsies. BrJDermatol 173(5):1239-1249

29. Mohammadpour I, Hatam GR, Handjani F et al (2019) Leishmania cytochrome b gene sequence polymorphismsinsouthernlran:relationships with different cutaneous clinical manifestations. BMC Infect Dis 19(1):98

\section{"Action-Fund" gegen Antibiotika-Resistenzen}

Um die weltweit steigenden Antibiotikaresistenzen einzudämmen, ist neben einem vernünftigen und zielgerichteten Einsatz der vorhandenen Wirkstoffe auch die Entwicklung neuer Antibiotika erforderlich. Der AMR "Action Fund" ist eine Initiative von 20 führenden biopharmazeutischen Unternehmen, die insgesamt mehr als eine Milliarde US-Dollar bis 2030 investieren um zwei bis vier neue Antibiotika zu entwickeln. Darüber hinaus sollte die öffentliche Hand in diesem Bereich in Forschung und Entwicklung investieren, stellt PHARMIG-Generalsekretär Alexander Herzog fest, denn die kostenintensive Entwicklung könne von Unternehmen aufgrund der durchaus sinnvollen
Strategie eines sparsamen Antibiotikaeinsatzes langfristig nicht refinanziert werden.

Die AMR Industry Alliance prognostiziert bei gleichbleibender Entwicklung der Antibiotikaresistenzen einen Anstieg der jährlichen Zahl der Todesopfer bis 2050 auf zehn Millionen Aktuell sterben weltweit pro Jahr bis zu 700.000 Menschen an den Folgen von Infektionen, bei denen Antibiotika keine Wirkung mehr zeigen. 
Hier steht eine Anzeige.

\section{曾 Springer}

